\title{
Counting the Number of Lithium Atoms in the Diffusion Channel by Annular Bright Field imaging.
}

\author{
Y. Oshima,* S. Lee**, H. Sawada***, F. Hosokawa***, E. Okunishi***, T. Kaneyama***, Y. \\ Kondo***, S. Niitaka****, H. Takagi****, Y. Tanishiro** and K. Takayanagi** $^{*}$ \\ * Research center for ultra HVEM, Osaka University, 7-1 Midorigaoka, Ibaraki, 567-0047, Japan \\ ** Department of Condensed Matter Physics, Tokyo Institute of Technology, 2-12-1-H-51 Oh- \\ okayama, Meguro-ku, Tokyo 152-8551, Japan \\ *** JEOL Ltd., 3-1-2 Musashino, Akishima, Tokyo 196-8558, Japan \\ **** Magnetic Materials Laboratory, RIKEN, 2-1 Hirosawa, Wako, Saitama 351-0198, Japan
}

The diffusion behavior of lithium ions is one of important issues to the development of lithium ion batteries (LIBs), because they determine the performance of LIBs. Recently, diffusion channel of lithium ions in an $\mathrm{LixFePO}_{4}$ crystal was visualized at the atomic scale by neutron diffraction [1], and the lithium diffusion behavior in same crystal was proposed as domino-cascade model by X-ray diffraction study [2]. Lithium ion diffusion has been found, by in-situ electron microscopy, to induce local structural and compositional changes in electrodes such as dendrite growth [3] The diffusion behavior was reported to be affected by the inhomogeneous distribution of the transition metal in composite materials, $\mathrm{Li}_{1.2} \mathrm{Mn}_{0.4} \mathrm{Fe}_{0.4} \mathrm{O}_{2}$ using electron energy loss spectroscopy [4].

Visualization of lithium atomic columns has been achieved by retrieving the exiting wave phase of a specimen from a focal series of high-resolution transmission electron microscopy images for $\mathrm{LiCoO}_{2}$ crystals [5] and $\mathrm{Al}_{3} \mathrm{Li}$ crystals [6]. Recently, direct imaging of lithium atomic columns in $\mathrm{LiV}_{2} \mathrm{O}_{4}$ [7] has been succeeded by annular bright field (ABF) imaging [8] with a scanning transmission electron microscopy (STEM). The ABF method has high sensitivity for imaging light elements (small $\mathrm{Z}$ number). The previous observations, however, were done for thick specimens [9], and the column intensity was independent of the specimen thickness.

Cowley has proposed a thin annular detector bright field imaging [10], which has similar configuration to $\mathrm{ABF}$ imaging, for improving spatial resolution. Based on phase object approximation, he showed that the image intensity at the atomic column is proportional to the projected potential. His study suggests that, for very thin specimen, the ABF imaging is a promising method to obtain the image intensity, which depends on the number of lithium atoms.

In this study, we demonstrate that the number of lithium atoms in the diffusion channel, which is the atomic columns along the [110] direction, was countable one-by-one from ABF image intensity. The lithium atoms in the diffusion channel of a single $\mathrm{LiV}_{2} \mathrm{O}_{4}$ crystal was observed by a sub-50 pm resolution STEM (R005) [11].

The wedge-shaped specimens of a single $\mathrm{LiV}_{2} \mathrm{O}_{4}$ crystal (a spinel structure, space group Fd-3m, $\mathrm{a}=0.824 \mathrm{~nm}$ ) were observed by ABF imaging from the [110] direction with R005 [11] at $300 \mathrm{kV}$. The specimens were very thin $(1-4 \mathrm{~nm})$. The probe beam had a convergent semi-angle of $30 \mathrm{mrad}$. The inner-outer semi-angle of the ABF detector was 20 - 30 mrad.

The ABF image in Fig. 1(a) shows dark dots at the positions of vanadium $\alpha(\mathrm{V} \alpha)$, vanadium $\beta$ $(\mathrm{V} \beta)$, oxygen, and lithium columns. The simulated image of the model reproduced the experimental image, as displayed in Fig. 1(b), in which all kinds of atomic columns including lithium columns appear as dark dots. The image intensity at all kinds of atomic columns decreased (darker) as the thickness increased in both experimental and the simulated images. We defined the height of the dip for $\mathrm{V} \alpha$ columns as the dip-height, $\mathrm{I}(V \alpha)$, in Fig. 1(c). As displayed in Fig.1(d), the dip-heights of $\mathrm{V} \alpha$ 
columns changed proportionally with the number of vanadium atoms in the column. It indicates that individual vanadium atoms in each atomic column gave a finite dip-height increase in the ABF image. Therefore, for other atomic columns, the dip-height was expected to increase in proportion with thickness increase. Fig. 2(a) is the enlarged ABF image in an area along the line C-C' of Fig.1(a). Their profile of Fig.2(b) shows the linear relationship between the thickness and the dipheight of lithium atomic column. Since the number of lithium atoms in the column increases one-byone from left to right- side in Fig. 2(a), the dip-hight of litium column is propotional to the number of lithium atoms. This result was also confirmed by analyzing the relationshipe bewteen the number of lithium atoms and the dip-height for about 140 lithium atomic columns. We concluded that the number of lithium atoms in the column was countable for very thin $\mathrm{LiV}_{2} \mathrm{O}_{4}$ specimen [12].

\section{References}

[1]S. Nishimura et al., Nat. Mater. 7 (2008) 707.

[2] C. Delmas et al., Nat. Mater. 7 (2008) 665.

[3] M. Morcrette et al., Nat. Mater. 2 (2003) 755.

[4] J. Kikkawa et al., Electrochem. Solid-State Lett. 11 (2008) A138.

[5] S.H.Yang S.H. et al., Nat. Mater. 2 (2003) 464.

[6] M. D. Rossell et al., Phys. Rev. B 80 (2009) 024110.

[7] Y. Oshima et al., J. Electron Microsc. 59 (2010) 457.

[8] E. Okunishi et al., Microsc. Microanal. 15 (Suppl 2) (2009) 164.

[9] S.D. Findlay et al., Appl. Phys. Lett. 95 (2009) 191913.

[10] J. M. Cowley, Ultramicroscopy 49 (1993) 4.

[11] H. Sawada et al., J. Elec. Micro. 58 (2009) 357.

[12] This work was supported by the Japan Science and Technology Agency (JST) under the CREST project.
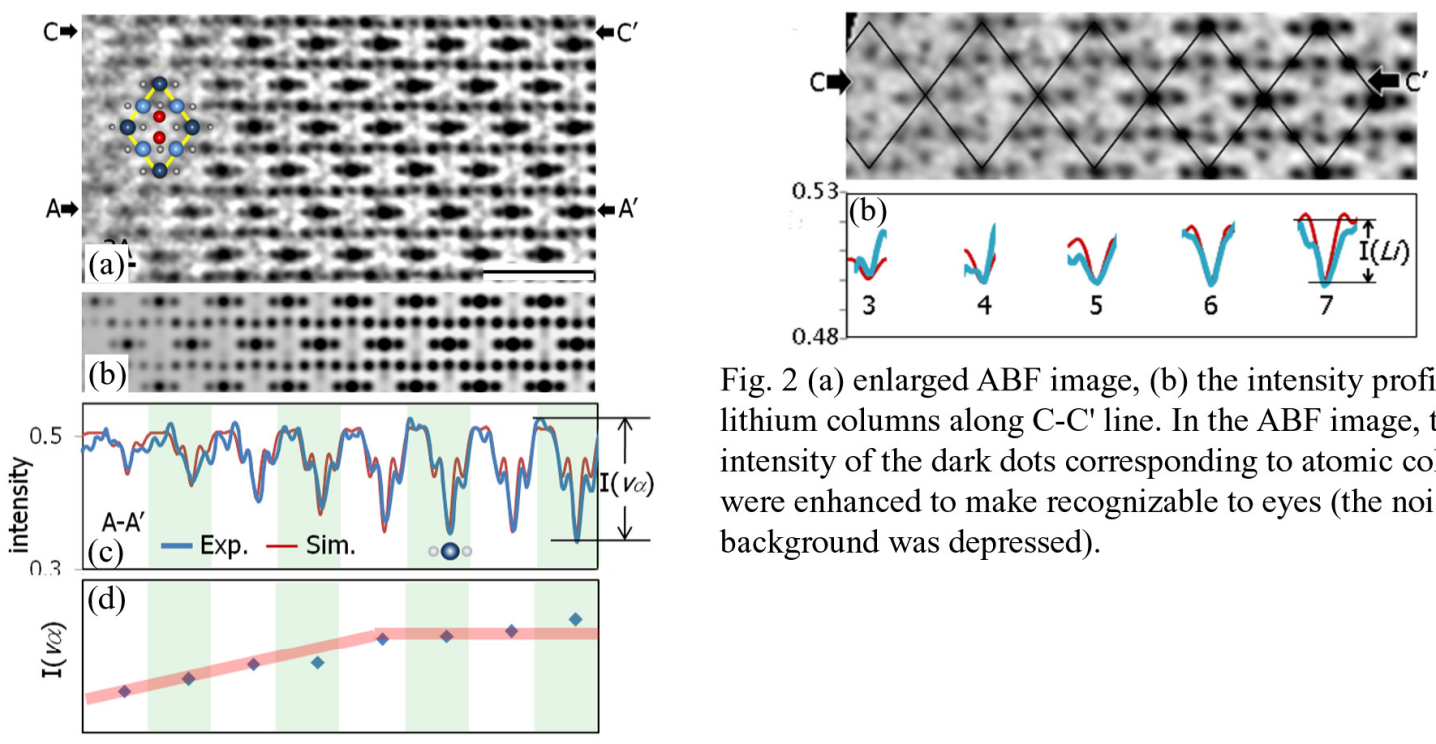

Fig. 2 (a) enlarged ABF image, (b) the intensity profile of lithium columns along $\mathrm{C}-\mathrm{C}^{\prime}$ line. In the $\mathrm{ABF}$ image, the intensity of the dark dots corresponding to atomic columns were enhanced to make recognizable to eyes (the noisy background was depressed).

Fig. 1 (a) a ABF image of wedge-shaped $\mathrm{LiV}_{2} \mathrm{O}_{4}$ specimen viewed from the [110] direction, (b) the simulated image, (c) the intensity profile along A-A' line, (d) the dip-height as a function of thickness. In the ABF image, rhombic lattice is the unit cell. Vanadium $\alpha(\mathrm{V} \alpha)$ columns are indicated by dark blue circles, vanadium $\beta\left(\mathrm{V}_{\beta}\right)$, light blue ones, oxygen, small ones and lithium, red ones. $\mathrm{V} \alpha$ column have two times the number of vanadium atoms than $\mathrm{V}_{\beta}$ one in the column when the thickness is the same. 